\title{
A Note on the Painlevé Property of Coupled KdV Equations
}

\author{
Sergei Sakovich \\ Institute of Physics, National Academy of Sciences of Belarus, 220072 Minsk, Belarus \\ Correspondence should be addressed to Sergei Sakovich; saks@tut.by
}

Received 25 October 2013; Accepted 9 January 2014; Published 19 February 2014

Academic Editor: Michael Grinfeld

Copyright (C) 2014 Sergei Sakovich. This is an open access article distributed under the Creative Commons Attribution License, which permits unrestricted use, distribution, and reproduction in any medium, provided the original work is properly cited.

We prove that one system of coupled KdV equations, claimed by Hirota et al. to pass the Painlevé test for integrability, actually fails the test at the highest resonance of the generic branch and therefore must be nonintegrable.

\section{Introduction}

In Section 6 of their paper [1], Hirota et al. reported that the system of coupled $\mathrm{KdV}$ equations

$$
\begin{array}{r}
\frac{\partial u_{i}}{\partial t}+6 a\left(\sum_{k=1}^{N} u_{k}\right) \frac{\partial u_{i}}{\partial x}+6(1-a) \\
\times\left(\sum_{k=1}^{N} \frac{\partial u_{k}}{\partial x}\right) u_{i}+\frac{\partial^{3} u_{i}}{\partial x^{3}}=0, \\
i=1,2, \ldots, N, \quad N \geq 2,
\end{array}
$$

passes the Painlevé test for integrability if and only if the parameter $a$ is equal to 1 , or $1 / 2$, or $3 / 2$. The authors of [1] pointed out that the cases $a=1$ and $a=1 / 2$ of (1) correspond to integrable systems of coupled $\mathrm{KdV}$ equations, whereas the problem of integrability of (1) with $a=3 / 2$ remains open.

In the present short note, we show that the system (1) with $a=3 / 2$ actually does not pass the Painlevé test, and its integrability should not be expected therefore.

\section{Singularity Analysis}

First of all, let us notice that the $N$-component system (1) can be studied in the form of the following triangular system of two coupled $\mathrm{KdV}$ equations:

$$
\begin{gathered}
v_{t}+6 v v_{x}+v_{x x x}=0 \\
w_{t}+6 a v w_{x}+6(1-a) w v_{x}+w_{x x x}=0
\end{gathered}
$$

where the new dependent variable $v$ is defined by $v=$ $\sum_{k=1}^{N} u_{k}$, the new dependent variable $w$ is any one of the $N$ components $u_{1}, \ldots, u_{N}$, and the subscripts $x$ and $t$ denote partial derivatives. Indeed, the system (1) is equivalent to the system consisting of the first equation of (2) along with $N-1$ copies of the second equation of (2) with $w=u_{1}, \ldots, u_{N-1}$ (say). Therefore, in order to check whether or not the system (1) passes the Painlevé test, it is sufficient to consider the two equations in (2) and do not repeat the same calculations for the remaining $N-2$ copies of the second equation of (2).

Setting $a=3 / 2$ in (2) and starting the Weiss-Kruskal algorithm of singularity analysis $[2,3]$, we use the expansions $v=v_{0}(t) \phi^{\alpha}+\cdots+v_{r}(t) \phi^{r+\alpha}+\cdots$ and $w=w_{0}(t) \phi^{\beta}+$ $\cdots+w_{r}(t) \phi^{r+\beta}+\cdots$ with $\phi_{x}(x, t)=1$ and determine branches (i.e., admissible choices of $\alpha, \beta, v_{0}, w_{0}$ ) together with corresponding positions $r$ of resonances (where arbitrary functions of $t$ can enter the expansions). The exponents $\alpha$ and $\beta$ and the positions of resonances turn out to be integer in all branches. In what follows, we only consider the generic singular branch, where $\alpha=\beta=-2, v_{0}=$ $-2, w_{0}(t)$ is arbitrary, and $r=-1,0,1,4,6,8$. This branch describes the singular behavior of generic solutions. There are also two nongeneric branches, but they correspond to the constraints $w_{0}=0$ and $w_{0}=w_{1}=0$ imposed on the generic branch and do not require any separate consideration therefore.

Substituting the expansions

$$
v=\sum_{n=0}^{\infty} v_{n}(t) \phi^{n-2}, \quad w=\sum_{n=0}^{\infty} w_{n}(t) \phi^{n-2}
$$


with $\phi_{x}(x, t)=1$ into the system (2) with $a=3 / 2$, we obtain the following recursion relations for the coefficients $v_{n}$ and $w_{n}$ of (3):

$$
\begin{aligned}
& (n-2)(n-3)(n-4) v_{n}+3(n-4) \\
& \quad \times \sum_{i=0}^{n} v_{i} v_{n-i}+(n-4) \phi_{t} v_{n-2}+v_{n-3, t}=0 \\
& (n-2)(n-3)(n-4) w_{n} \\
& +3 \sum_{i=0}^{n}(3 n-4 i-4) v_{i} w_{n-i}+(n-4) \phi_{t} w_{n-2}+w_{n-3, t}=0, \\
& \quad n=0,1,2,3, \ldots,
\end{aligned}
$$

where the subscript $t$ denotes the derivative with respect to $t$, and $v_{k}=w_{k}=0$ for $k=-3,-2,-1$ formally.

Now we have to check whether the recursion relations (4) are compatible at the resonances.

The resonance -1 , as always, corresponds to the arbitrariness of the function $\psi$ in $\phi=x+\psi(t)$.

We have $v_{0}=-2$ in (4) at $n=0$, for the chosen branch. The function $w_{0}(t)$ remains arbitrary, which corresponds to the resonance 0 .

Setting $n=1$ in (4), we find that $v_{1}=0$, while the function $w_{1}(t)$ remains arbitrary, and the compatibility condition at the resonance 1 is satisfied.

At $n=2$ and $n=3$, which are not resonances, we get from (4), respectively,

$$
\begin{gathered}
v_{2}=-\frac{1}{6} \phi_{t}, \quad w_{2}=\frac{1}{12} w_{0} \phi_{t}, \\
v_{3}=0, \quad w_{3}=\frac{1}{60} w_{1} \phi_{t}+\frac{1}{30} w_{0, t} .
\end{gathered}
$$

Setting $n=4$ in (4), we find that

$$
w_{4}=-\frac{1}{2} v_{4} w_{0}+\frac{1}{48} w_{1, t},
$$

while the function $v_{4}(t)$ remains arbitrary, and the compatibility condition at the resonance 4 is satisfied.

At $n=5$, which is not a resonance, we find from (4) that

$$
\begin{gathered}
v_{5}=-\frac{1}{36} \phi_{t t} \\
w_{5}=-\frac{1}{4} v_{4} w_{1}-\frac{1}{7200} w_{1} \phi_{t}^{2}+\frac{1}{900} w_{0, t} \phi_{t}+\frac{1}{72} w_{0} \phi_{t t} .
\end{gathered}
$$

Setting $n=6$ in (4), we obtain

$$
w_{6}=-\frac{1}{2} v_{6} w_{0}-\frac{1}{14400} w_{1, t} \phi_{t}+\frac{31}{3600} w_{1} \phi_{t t}+\frac{1}{1800} w_{0, t},
$$

while the function $v_{6}(t)$ remains arbitrary, and the compatibility condition at the resonance 6 is satisfied.
At $n=7$, which is not a resonance, we get from (4) the following:

$$
\begin{gathered}
v_{7}=-\frac{1}{24} v_{4, t} \\
w_{7}=-\frac{1}{2} v_{6} w_{1}+\frac{17}{1680} v_{4} w_{1} \phi_{t}+\frac{1}{201600} w_{1} \phi_{t}^{3}+\frac{1}{48} v_{4, t} w_{0} \\
-\frac{1}{105} v_{4} w_{0, t}-\frac{1}{25200} w_{0, t} \phi_{t}^{2}+\frac{1}{2016} w_{1, t t}
\end{gathered}
$$

The highest resonance in the chosen branch is 8 . Setting $n=8$ in the recursion relations (4), we find that

$$
v_{8}=-\frac{1}{6} v_{4}^{2}+\frac{1}{2592} \phi_{t t t}
$$

the function $w_{8}(t)$ remains arbitrary, but the compatibility condition at the resonance 8 is not satisfied, and we obtain the following constraint imposed on some of arbitrary functions that appeared at lower resonances:

$$
300 v_{4, t} w_{1}-7 w_{1} \phi_{t} \phi_{t t}+6 w_{0, t} \phi_{t t}=0
$$

The appearance of the constraint (11) means that the Laurent type expansions (3) do not represent the general solution of the studied system, and we have to modify the expansion for $w$ by introducing logarithmic terms, starting from the term proportional to $\phi^{6} \log \phi$. This nondominant logarithmic branching of solutions is a clear symptom of nonintegrability. Consequently, the case $a=3 / 2$ of the system (2) - and of the system (1), equivalently_fails the Painlevé test.

\section{Conclusion}

We have shown that, contrary to the claim of Hirota et al. [1], the system of coupled KdV equations (1) with $a=3 / 2$ does not pass the Painlevé test for integrability.

Let us note, moreover, that the singularity analysis of coupled $\mathrm{KdV}$ equations has been addressed in the papers $[4,5]$, published prior to [1]. In particular, the integrable cases $a=1$ and $a=1 / 2$ of the system (1) can be found in [5] as the systems (vi) and (vii), respectively, which have passed the Painlevé test, whereas the case $r_{1}=1$ in Section 2.1.3 of [5] predicts that the system (1) with $a=3 / 2$ must fail the Painlevé test for integrability.

The obtained result that the system of coupled $\mathrm{KdV}$ equations (1) with $a=3 / 2$ actually does not pass the Painlevé test for integrability explains very well why no Lax representation has been proposed as yet for this case of coupled KdV equations.

\section{Conflict of Interests}

The author declares that there is no conflict of interests regarding the publication of this paper. 


\section{Acknowledgment}

The author is grateful to the anonymous reviewer for valuable comments.

\section{References}

[1] R. Hirota, X.-B. Hu, and X.-Y. Tang, "A vector potential KdV equation and vector Ito equation: Soliton solutions, bilinear Bäcklund transformations and Lax pairs," Journal of Mathematical Analysis and Applications, vol. 288, no. 1, pp. 326-348, 2003.

[2] J. Weiss, M. Tabor, and G. Carnevale, “The Painlevé property for partial differential equations," Journal of Mathematical Physics, vol. 24, no. 3, pp. 522-526, 1982.

[3] M. Jimbo, M. D. Kruskal, and T. Miwa, "Painlevé test for the self-dual Yang-Mills equation," Physics Letters A, vol. 92, no. 2, pp. 59-60, 1982.

[4] A. (Kalkanli) Karasu, "Painlevé classification of coupled Korteweg-de Vries systems," Journal of Mathematical Physics, vol. 38, no. 7, pp. 3616-3622, 1997.

[5] S. Y. Sakovich, "Coupled KdV equations of Hirota-Satsuma type," Journal of Nonlinear Mathematical Physics, vol. 6, no. 3, pp. 255-262, 1999. 


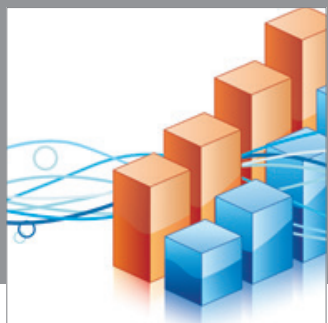

Advances in

Operations Research

mansans

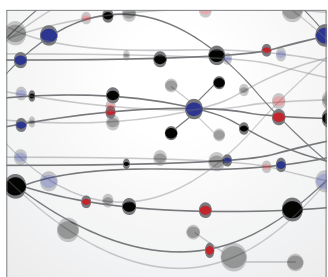

The Scientific World Journal
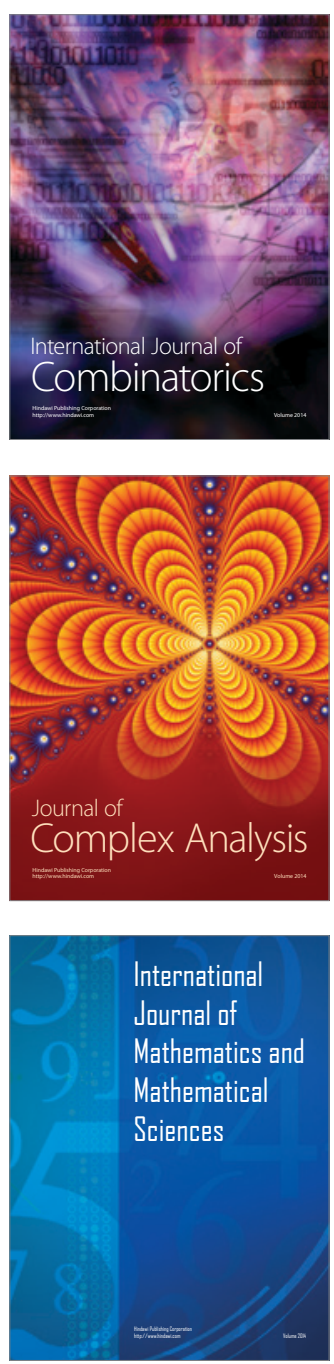
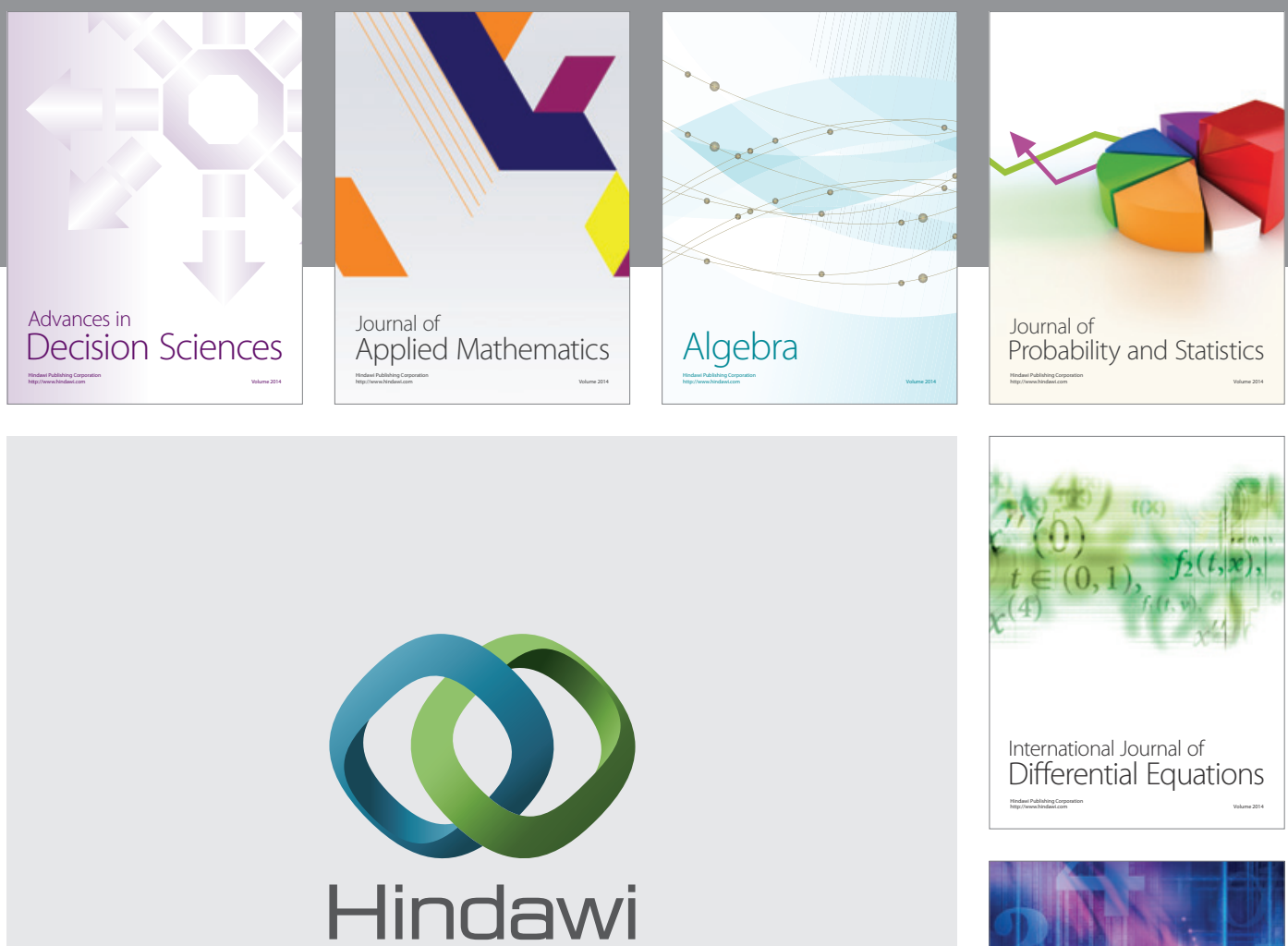

Submit your manuscripts at http://www.hindawi.com
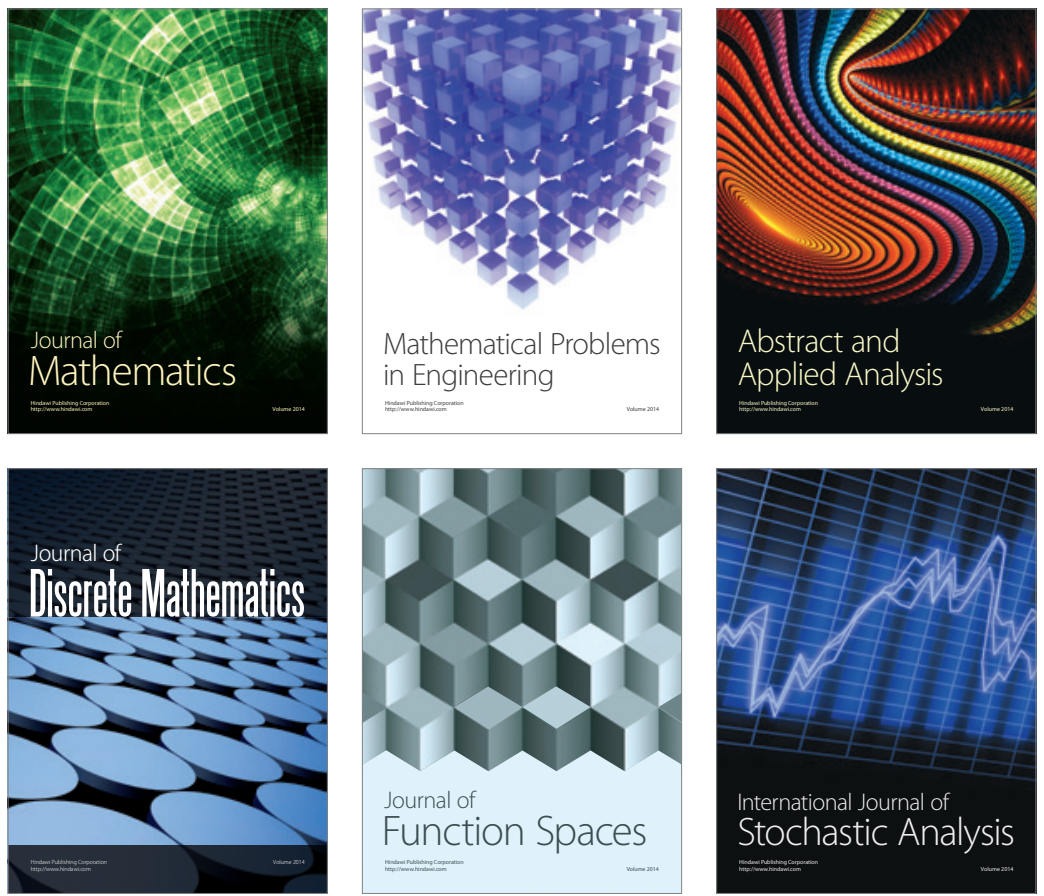

Journal of

Function Spaces

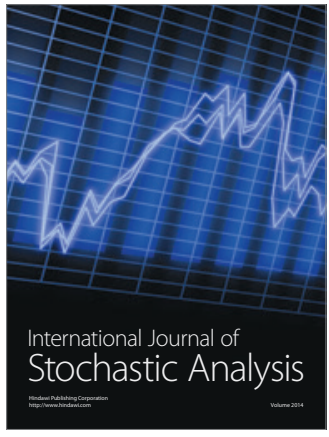

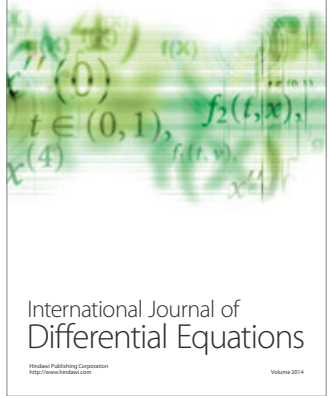
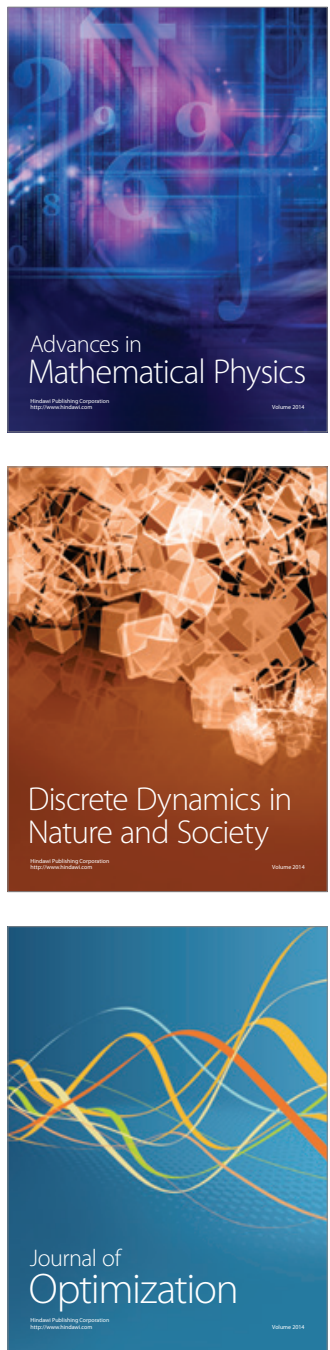